\title{
Intermittent Hypoxia Disrupts Glucose Homeostasis in Liver Cells in an Insulin- Dependent and Independent Manner
}

\author{
Chen Juan Gua Hua Hua Yia Jing Feng ${ }^{a} \quad$ Zhi Guo Zhang ${ }^{b} \quad$ Jun Zhou \\ Li Na Zhou ${ }^{a}$ Jian Ping Zhou ${ }^{a}$ Min Lia Qing Yun Lia
}

${ }^{a}$ Department of Respiratory and Critical Care Medicine, Ruijin Hospital, Shanghai Jiao Tong University School of Medicine, bepartment of Endocrinology and Metabolism, Ruijin Hospital, Shanghai Jiao Tong University School of Medicine, China

\section{Key Words}

Intermittent hypoxia $\cdot$ Liver cells $\cdot$ Glucose homeostasis $\cdot$ Forkhead box protein O1 • C-Jun $\mathrm{NH}_{2}$-terminal-kinase

\begin{abstract}
Background/Aims: Obstructive sleep apnea is associated with diabetes and insulin resistance, but the underlying mechanisms remain unclear. The purpose of the current study was to determine the molecular effects of intermittent hypoxia (IH) on hepatic insulin signaling and glucose homeostasis, and whether c-Jun $\mathrm{NH}_{2}$-terminal-kinase (JNK) contributed to metabolic responses to $\mathrm{IH}$ in liver cells. Methods: The human $\mathrm{HepG}_{2}$ cells and rat FAO cells were exposed to $10,30,120,240$ or 360 cycles of $\mathrm{IH}\left(1 \% \mathrm{O}_{2}\right.$ for $60 \mathrm{~s}$ followed by $21 \% \mathrm{O}_{2}$ for $60 \mathrm{~s}, 7.5$ cycles per hour) or normoxia as a control. In a subgroup, we exposed cells to 360 cycles of IH with the JNK inhibitor SP600125. After IH exposure, cell glycogen content and glucose output were measured using colorimetric assay kits. Canonical insulin signaling and gluconeogenic genes were measured by western blot and quantitative polymerase chain reaction. Results: IH decreased insulin-stimulated protein kinase B (AKT) / glycogen synthase

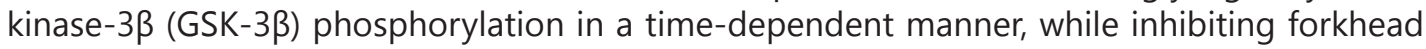
box protein $\mathrm{O} 1$ (FOXO1) expression and phosphoenolpyruvate carboxykinase (PEPCK) transcription independent of insulin signaling. JNK inhibitor SP600125 partially restored AKT/ GSK-3 $\beta$ phosphorylation and glycogen synthesis, but did not affect other $\mathrm{IH}$-induced glucose metabolic changes. Conclusion: IH in vitro impaired insulin signal transduction in liver cells as assessed by inhibited AKT/GSK-3 $\beta$ phosphorylation via JNK activation. IH inhibited FOXO1 and gluconeogenesis in an insulin-independent manner.

(C) 2018 The Author(s)

Published by S. Karger AG, Basel
\end{abstract}

\section{Introduction}

Obstructive sleep apnea (OSA) is associated with increased risk of diabetes [1, 2] and insulin resistance (IR) [3, 4]. The mechanisms underlying this association remain unclear.

Dr. Qing Yun Li and Dr. Min Li

\section{KARGER}

Department of Respiratory and Critical Care Medicine, Ruijin Hospital, Shanghai Jiao Tong University School of Medicine; No.197, Ruijin Er Road, Shanghai, 200025, (China)

Tel.86-21-64370045-680803, E-Mail liqingyun68@hotmail.com, limin_4212@163.com 
Intermittent hypoxia (IH), one of the cardinal pathophysiologic features in OSA, has the potential to facilitate tissue oxygen fluctuations [5, 6], trigger cell stress responses [7, 8], and lead subsequently to cell dysfunction. The liver plays an essential role in metabolic homeostasis and is a major insulin target organ [9]. In a rodent model of IH simulating OSA, the liver exhibited the highest amplitude of swings of oxygen partial pressure compared to muscle and fat [5]. Hence, the liver is especially vulnerable to $\mathrm{IH}$, and liver metabolic dysfunction may contribute to systemic cardio-metabolic disorders in OSA patients.

Human and animal studies support the detrimental effects of IH on glucose metabolism and whole-body insulin sensitivity [10-12]. Several studies evaluated liver glucose output and insulin signal transduction in response to IH [11-14], but did not achieve consistent conclusions. In terms of how IH influences hepatic glucose metabolism and insulin sensitivity, the major proposed mechanism is the systemic activation of sympathetic nervous system in response to $\mathrm{IH}[11,13,15]$. Less attention has been directed towards direct effects of IH on liver cells per se. Furthermore, the impact of insulin on liver metabolism can occur via both direct (binding to hepatocytes) and indirect (suppression of pancreatic glucagon secretion, adipose tissue lipolysis) pathways [16]. In this study, we use an in vitro cell model to examine the impacts of $\mathrm{IH}$ on direct insulin signaling in human $\mathrm{HepG}_{2}$ cells.

In the liver, insulin reduces glucose production by down-regulating both gluconeogenesis and glycogenolysis [17]. Failure of insulin to suppress hepatic glucose production contributes to fasting hyperglycemia, a hallmark of type 2 diabetes. $\mathrm{HepG}_{2}$ cell line was established from a human hepatoma biopsy specimen and is known to resemble differentiated hepatocytes [18, 19]. FAO cells, derived from the rat H4IIE hepatoma cell line, are suitable for the evaluation of glucose production as these cells possess a complete gluconeogenic enzyme system that allows them to survive and grow in low-glucose or glucose-free medium [20]. In this study, we focused on two insulin-signaling pathways in $\mathrm{HepG}_{2}$ cells and examined glucose output in FAO cells. First, insulin signals through its receptor, insulin receptor substrates and phosphoinositide 3' - kinase (PI3K) to activate the serine/threonine kinase protein kinase B (AKT). AKT phosphorylates and inactivates glycogen synthase kinase (GSK)-3, thus increasing glycogen synthase activity and stimulating glycogen synthesis [21]. Second, insulin regulates the transcription factor forkhead box protein 01 (FOX01) which promotes the expression of phosphoenolpyruvate carboxykinase (PEPCK) and glucose 6-phosphatase (G6Pase), two key gluconeogenic genes [22]. c-Jun $\mathrm{NH}_{2}$-terminal-kinase (JNK) is an established cause of intracellular insulin resistance [23]. JNK interferes with insulin signaling by inhibitory serine phosphorylation of insulin receptor substrate-1 (IRS-1) [24, 25]. We hypothesized that JNK activation in response to IH contributes to impaired insulin signaling in liver cells. To test our hypothesis, we exposed $\mathrm{HepG}_{2}$ and FAO cells to in vitro IH and examined 1) canonical insulin signal transduction; 2) regulation of hepatic glycogen synthesis and gluconeogenesis; 3) JNK activation and its role in IH-related hepatic IR.

\section{Materials and Methods}

\section{Cell Culture and Treatment}

The human $\mathrm{HepG}_{2}$ and rat FAO cell lines were obtained from cell banks of Shanghai Institutes of Endocrine and Metabolic Diseases, Ruijin Hospital, Shanghai Jiao Tong University School of Medicine (China). Cells were cultured in Dulbecco's Modified Eagle's medium (DMEM) supplemented with 10\% fetal bovine serum (FBS), $2 \mathrm{mM} \mathrm{L-glutamine,} \mathrm{100U/mL} \mathrm{Penicillin} \mathrm{and} 100 \mu \mathrm{g} / \mathrm{mL}$ Streptomycin (Gibco; Thermo Fisher Scientific, Waltham, MA, USA) in an atmosphere of $5 \% \mathrm{CO}_{2}$ at $37{ }^{\circ} \mathrm{C}$. Prior to all experiments, cells were refreshed with $2 \mathrm{ml}$ culture medium. In experiments involving treatment with JNK inhibitor, $\mathrm{HepG}_{2}$ cells were preincubated for $2 \mathrm{~h}$ with either SP600125 $10 \mu \mathrm{mol} / \mathrm{L}$ (Sigma-Aldrich, St. Louis, Missouri, USA) or DMSO (vehicle). In experiments involving insulin signal transduction, $\mathrm{HepG}_{2}$ cells were treated with insulin $100 \mathrm{nmol} / \mathrm{L}$ (Humulin R, Eli Lilly and Company, IN, USA) or PBS (Invitrogen, Carlsbad, CA, USA) for 30min before the end of experiments (IH or control).

\section{KARGER}




\section{Cellular Physiology Cell Physiol Biochem 2018;47:1042-1050 \begin{tabular}{l|l} 
and Biochemistry Published $10.1159 / 000490169$ & $\begin{array}{l}\text { DO } 2018 \text { The Author(s). Published by S. Karger AG, Basel } \\
\text { www.karger.com/cpb }\end{array}$
\end{tabular}}

Gu et al.: Intermittent Hypoxia and Hepatic Glucose Homeostasis

Intermittent Hypoxia

A controlled gas delivery system was designed to regulate the flow of nitrogen and air into a $\mathrm{CO}_{2}$ incubator (Galaxy 14s, New Brunswick Scientific, Edison, NJ, USA) over a defined and repeatable profile with $\mathrm{O}_{2}$ levels monitored by an electrode placed in the incubator as described previously [26]. During each cycle of $\mathrm{IH}, \mathrm{O}_{2}$ level was reduced from $21 \%$ to $1 \%$ over $160 \mathrm{~s}$, kept at $1 \%$ for 60 s, then reoxygenated to $21 \%$ over $200 \mathrm{~s}$ and kept at $21 \%$ for $60 \mathrm{~s}$, resulting in 7.5 hypoxic events per hour. Cell cultures were exposed to $10,30,120,240$ or 360 cycles of IH at $37^{\circ} \mathrm{C}$. For control cells, $\mathrm{O}_{2}$ levels were maintained at $21 \%$.

Glycogen Assay

During the last $5.5 \mathrm{~h}$ of the experiments (IH or control), $\mathrm{HepG}_{2}$ cell culture medium was changed to DMEM containing $0.5 \%$ bovine serum albumin (BSA) (Invitrogen) for $4 \mathrm{~h}$. Then cells were incubated with DMEM containing high glucose levels in the presence of insulin $100 \mathrm{nmol} / \mathrm{L}$ (Humulin R) for 90min. After washing cultured cells three times with ice-cold PBS, we measured glycogen from cell lysates using a Glycogen Colorimetric Assay Kit II (Biovision, Inc., Milpitas, CA, USA) according to the manufacturer's instructions. All samples were assessed in duplicate and normalized to $\mu \mathrm{g}$ of protein as determined by bicinchoninic acid assay (BCA protein assay, Thermo Scientific, Waltham, MA, USA).

\section{Measurement of Glucose Output}

During the last $6 \mathrm{~h}$ of the experiments (IH or control), FAO cell culture medium was changed to DMEM containing $20 \mathrm{mmol} / \mathrm{L}$ sodium lactate and $2 \mathrm{mmol} / \mathrm{L}$ sodium pyruvate (Sigma-Aldrich, St. Louis, MO, USA) without glucose or phenol red. After $6 \mathrm{~h}$, glucose contents in the supernatant were measured by a Glucose Oxidase Colorimetric Assay Kit (Sigma-Aldrich, St. Louis, MO, USA). All samples were assessed in duplicate and normalized to $\mu \mathrm{g}$ of cellular protein as determined by bicinchoninic acid assay (BCA protein assay, Thermo Scientific, Waltham, MA, USA).

\section{Western Blot Analyses}

The total protein extracts were isolated from $\mathrm{HepG}_{2}$ cell lysates for analysis. Western blotting was performed using primary antibodies against phosphorylated AKT, AKT, phosphorylated GSK-3 $\beta$, GSK-3 $\beta$, phosphorylated FOX01, FOX01, phosphorylated JNK, JNK, $\beta$-actin (Cell Signaling Technology, Danvers, MA, USA), heat shock protein 90 (HSP90) (Santa Cruz Biotechnology, Santa Cruz, CA, USA), and appropriate horseradish peroxidase (HRP)- conjugated secondary antibody (Cell Signaling Technology). Western blots were developed using enhanced chemiluminescence (ECL) western blotting substrate (Thermo Fisher Scientific) and quantified using Adobe Photoshop CS6 (San Jose, CA, USA).

\section{Quantitative Polymerase Chain Reaction (qPCR)}

Total RNA was prepared from $\mathrm{HepG}_{2}$ cell lysates using Trizol reagent (Invitrogen). First-strand cDNA was synthesized from total RNA using the Reverse Transcription System (A3500, Promega, Madison, WI, USA). Gene expression was analyzed by real-time PCR with $2^{-\Delta \Delta \mathrm{Ct}}$ relative quantitative method with the Applied Biosystems ${ }^{\mathrm{TM}}$ QuantStudio $^{\mathrm{TM}} 6$ Flex Real-Time PCR Instrument (Thermo Fisher Scientific). Gene expression levels were normalized to housekeeping gene $\beta$-actin. The results were expressed as relative fold changes to controls. The forward and reverse primers of genes were listed as follows. PCK2: F: 5'-GGCTGAGAATACTGCCACACT-3'; R: 5'-ACCGTCTTGCTCTCTACTCGT-3'; G6PC: F: 5'CTACTACAGCAACACTTCCGTG-3'; R: 5'- GGTCGGCTTTATCTTTCCCTGA-3'.

\section{Statistical Analyses}

Statistical analyses were performed using STATA version 14.0 (StataCorp LLC, USA). All the data were normally distributed and presented as mean \pm standard deviation (SD). Comparisons of means between two groups were performed using two-tailed paired student's t test. Comparisons among 3 or more groups were conducted by one-way analysis of variance (ANOVA) and Bonferroni test for post hoc multiple comparisons. $P<0.05$ was considered as statistically significant.

\section{Results}

IH inhibited insulin-stimulated AKT/GSK-3 $\beta$ signaling in a time-dependent manner

Insulin binding to its receptor initiates a signaling cascade that begins with IRS-1 phosphorylation, PI3K activation, and phosphorylation of AKT. AKT phosphorylation leads 
Gu et al.: Intermittent Hypoxia and Hepatic Glucose Homeostasis

to the phosphorylation of GSK-3 $\beta$ (p-GSK3 $\beta$ ), which promotes glycogen synthesis. Insulinstimulated phosphorylation of AKT (p-AKT) and GSK-3 $\beta$ (p-GSK3 $\beta$ ) in $\mathrm{HepG}_{2}$ cells was measured after 10, 30, 120, 240 or 360 cycles of IH. Expression of p-AKT and p-GSK3 $\beta$ decreased significantly in a time-dependent manner, while total AKT and GSK-3 $\beta$ remained stable during IH exposure (Fig. 1 A-D). These changes in insulin transduction would be expected to reduce glycogen synthesis in response to insulin. However, we did not observe significant changes in hepatic glycogen content after IH exposure (Fig. $1 \mathrm{E}$ ).

IH inhibited FOXO1 and phosphoenolpyruvate carboxykinase (PEPCK) independent of insulin signaling

AKT activation also increases FOX01 phosphorylation, which further enhances insulin's regulation of intracellular glucose metabolism. In the un-phosphorylated state, FOXO1 remains in the nucleus and upregulates transcription of G6Pase and PEPCK, two key enzymes involved in gluconeogenesis. FOXO1 phosphorylation by AKT causes its nuclear exclusion and inactivation. Thus, insulin suppresses hepatic glucose production through a decrease in G6Pase and PEPCK transcription activity [27]. In HepG ${ }_{2}$ cells, IH reduced FOXO1

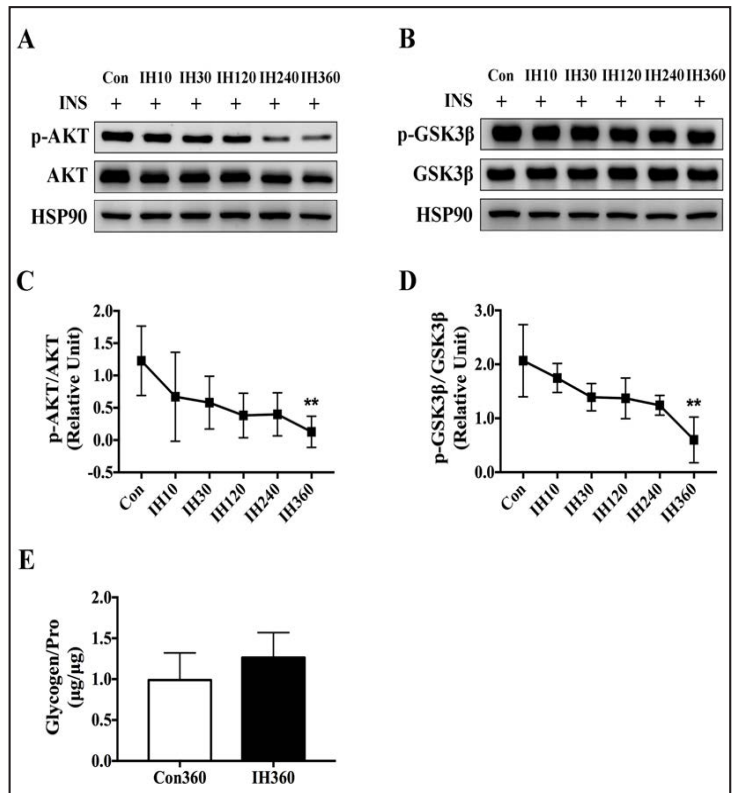

Fig. 1. IH inhibited insulin-stimulated AKT/GSK-3 $\beta$ signaling in a time-dependent manner. The protein expression of AKT, GSK-3 $\beta$, HSP90, and phosphorylation states of AKT and GSK-3 $\beta$ were determined by Western blot in $\mathrm{HepG}_{2}$ cells $(\mathrm{A}, \mathrm{B})$. The phosphor-to-total ratios were calculated and expressed in relative unit $(C, D)$, $n=6$ for each group. (E) Glycogen contents in $H_{e p} G_{2}$ cells after 360 cycles of IH or control. The results were adjusted by sample protein concentration, $\mathrm{n}=5$ for each group. Data are means $\pm \mathrm{SD}$. ${ }^{* *} \mathrm{P}<0.01$ versus control cells.

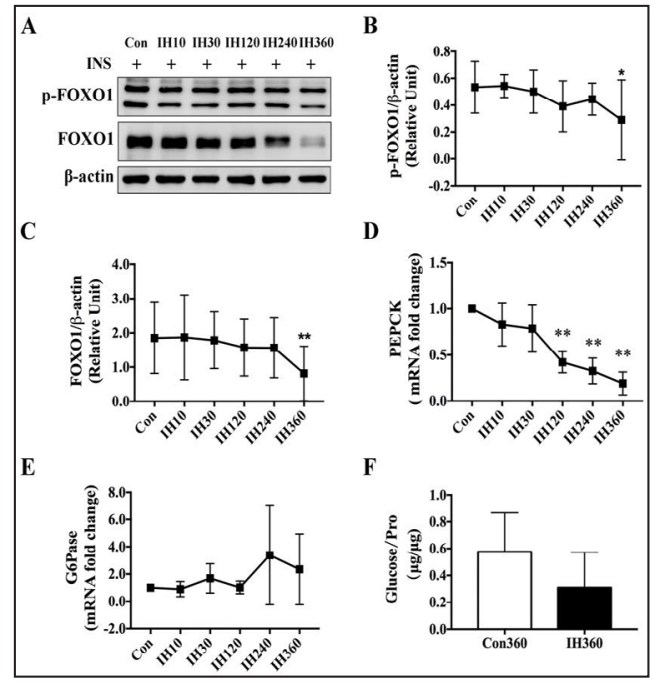

Fig. 2. IH inhibited FOXO1 and PEPCK independent of insulin signaling. The protein expression of FOX01, $\beta$-actin, and phosphorylation states of FOX01 were determined by Western blot in $\mathrm{HepG}_{2}$ cells (A). FOXO1 to $\beta$-actin and phosphorylated FOXO1 to $\beta$-actin ratio were calculated and expressed in relative unit, $n=6$ for each group $(B, C)$. Gene expression levels of PEPCK and G6Pase in $\mathrm{HepG}_{2}$ cells were analyzed by real-time PCR that were normalized to housekeeping gene $\beta$-actin. The results were expressed as relative fold changes to control cells, $\mathrm{n}=4$ for each group (D, E). (F) Glucose output of FAO cells after 360 cycles of IH or control. The results were adjusted by cellular protein concentration, $\mathrm{n}=8$ for each group. Data are means $\pm \mathrm{SD}$. ${ }^{*} \mathrm{P}<0.05,{ }^{* *} \mathrm{P}<0.01$ versus control cells. 
phosphorylation in accordance with reduced AKT/GSK-3 $\beta$ phosphorylation. Unexpectedly, IH also decreased total FOXO1 expression (Fig. 2 A-C). In parallel, IH inhibited transcription of PEPCK in a time-dependent manner (Fig. 2 D), while G6Pase was not affected (Fig. 2 E). Thus, IH inhibited FOXO1 expression and phosphorylation, and the net effect on its target genes was the suppression of PEPCK transcription. We further measured glucose production in FAO cells after IH exposure, which appeared to be lower than control cells, but did not achieve statistical difference (Fig. 2 F, $P=0.087$ ).

IH activated JNK signaling which contributed to the inhibition of insulin-stimulated AKT/ GSK-3 $\beta$ phosphorylation and glycogen synthesis.

In the setting of obesity and inflammation, activation of JNK directly interferes with insulin signaling by serine phosphorylation of IRS-1, which blocks the downstream insulin signal transduction through PI3K-AKT $[24,25]$. To determine whether IH-induced changes in insulin signaling and glucose metabolism are mediated by JNK activation, we examined phosphorylation of JNK after 10, 30, 120, 240 or 360 cycles of IH in $\mathrm{HepG}_{2}$ cells. As expected, JNK phosphorylation increased significantly after 360 cycles of IH (Fig. 3 A, B). Secondly, we pretreated a subset of $\mathrm{HepG}_{2}$ cells with JNK inhibitor (SP600125 $10 \mu \mathrm{mol} / \mathrm{L}$ ), or DMSO (vehicle) $2 \mathrm{~h}$ before IH exposure. JNK phosphorylation, basal and insulin-stimulated AKT/ GSK-3 $\beta / F O X 01$ signaling were measured after 360-cycle IH exposure. With SP600125 pretreatment, we no longer observed IH-induced phosphorylation of JNK (Fig. 3 C, D).

In terms of insulin signaling, insulin increased phosphorylation of AKT and GSK-3 $\beta$ as expected in control groups. 360-cycle IH inhibited insulin-stimulated phosphorylation of AKT and GSK-3 $\beta$, but did not affect their basal levels. Total AKT and GSK-3 $\beta$ remained stable during IH exposure. SP600125 pretreatment partially restored AKT/GSK-3 $\beta$ phosphorylation in the context of IH (Fig. 4 A, C, D). Furthermore, glycogen content was significantly higher in the IH group than in the control group, with SP600125 pretreatment (Fig. 4 B). Regarding FOX01 signaling, insulin stimulated FOX01 phosphorylation in control groups. 360-cycle IH decreased total and phosphorylated FOXO1 expression both in the absence and presence of insulin. We did not find any improvement in p-FOXO1/ FOXO1 signaling with SP600125 pretreatment after IH exposure (Fig. 5).

\section{Discussion}

OSA is characterized by repetitive upper airway obstruction during sleep leading to intermittent hypoxia (IH) and sleep fragmentation. The liver is more prone to local fluctuations in oxygen partial pressure than muscle or adipose tissue [5]. Our previous study revealed that $\mathrm{IH}$ inhibited AKT phosphorylation in response to insulin in $\mathrm{HepG}_{2}$ cells, which was partially

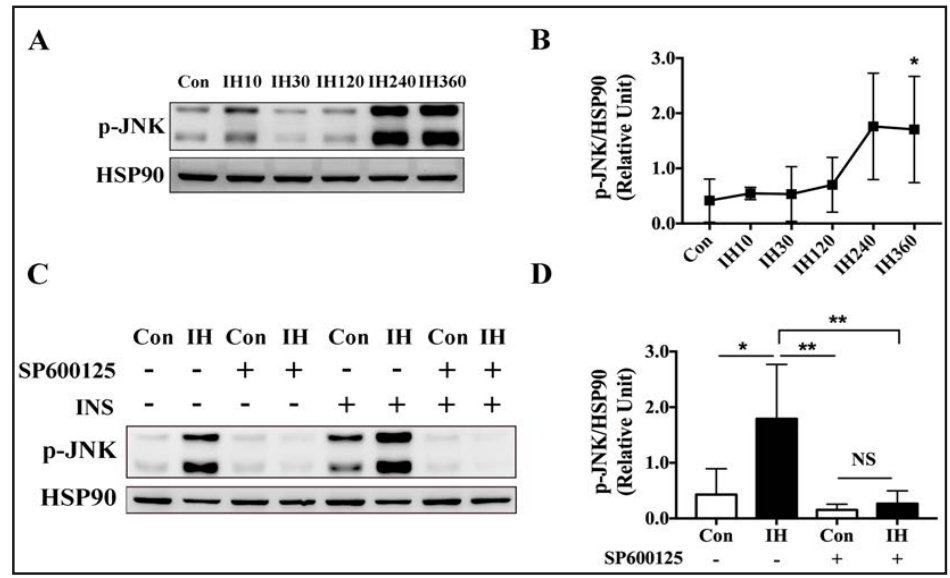

Fig. 3. IH activated JNK signaling which was abolished by SP600125 pretreatment. HepG $\mathrm{G}_{2}$ cells were exposed to $10,30,120,240,360$ cycles of IH or control. The protein expression of HSP90, and phosphorylation states of JNK were determined by Western blot (A). Phosphorylated JNK to HSP90 ratio was calculated and expressed in relative unit, n $=8$ for each group (B). A subset of $\mathrm{HepG}_{2}$ cells was pretreated with JNK inhibitor, or DMSO $2 \mathrm{~h}$ before 360 -cycle IH exposure. The protein expression of HSP90, and phosphorylation states of JNK in the absence and presence of insulin were determined by Western blot (C). Phosphorylated JNK to HSP90 ratio was calculated and expressed in relative unit, $\mathrm{n}=5$ for each group (D). Data are means \pm SD. ${ }^{*} \mathrm{P}<0.05$, $* * \mathrm{P}<0.01$ between groups. 


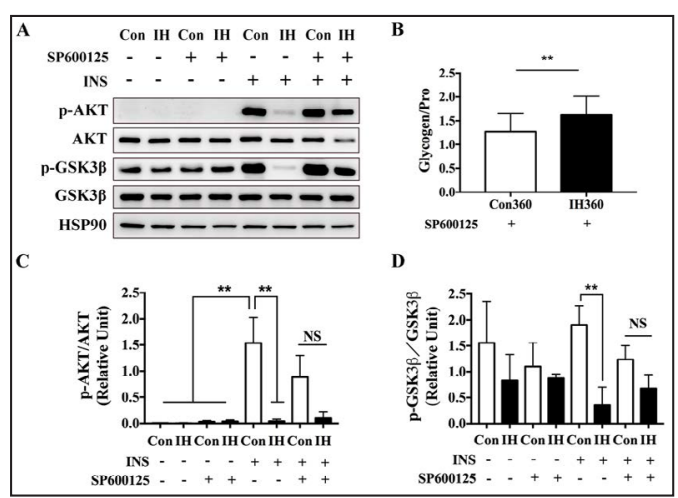

Fig. 4. JNK activation contributed to the inhibition of insulin-stimulated AKT/GSK-3 $\beta$ phosphorylation and glycogen synthesis in the context of IH. HepG $\mathrm{H}_{2}$ cells were pretreated with JNK inhibitor, or DMSO (vehicle) $2 \mathrm{~h}$ before 360-cycle IH or control. The protein expression of AKT, GSK-3 $\beta$, HSP90, and phosphorylation states of AKT and GSK-3 $\beta$ in the absence and presence of insulin were determined by Western blot (A). Glycogen contents were measured and compared between IH and control cells with SP600125 pretreatment. The results were adjusted by sample protein concentration, $\mathrm{n}=5$ for each group (B). The phosphor-to-total ratios of AKT and GSK$3 \beta$ were calculated and expressed in relative unit, $\mathrm{n}=6$ for each group (C, D). Data are means \pm SD. $* * \mathrm{P}<0.01$ between groups.

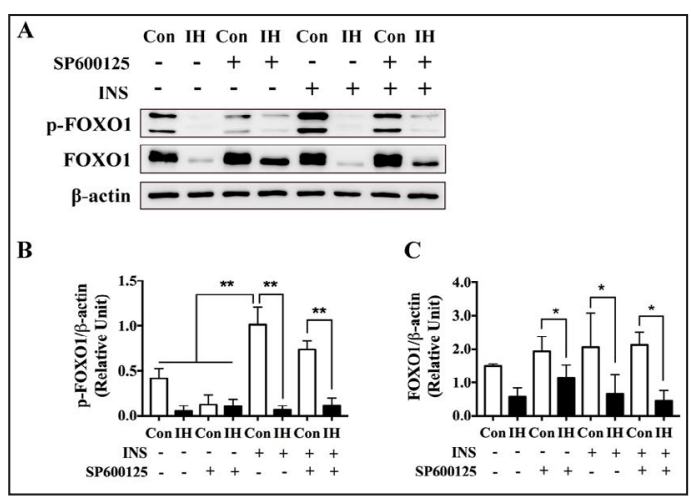

Fig. 5. JNK activation had no impact on FOXO1 signaling in the context of $\mathrm{IH}$. HepG $\mathrm{G}_{2}$ cells were pretreated with JNK inhibitor, or DMSO (vehicle) $2 \mathrm{~h}$ before 360 -cycle IH or control. The protein expression of FOX01, $\beta$-actin, and phosphorylation states of FOXO1 were determined by Western blot (A). FOX01 to $\beta$-actin and phosphorylated FOXO1 to $\beta$-actin ratio were calculated and expressed in relative unit, $\mathrm{n}=6$ for each group $(\mathrm{B}, \mathrm{C})$. Data are means $\pm \mathrm{SD} .{ }^{*} \mathrm{P}<0.05$ between groups, ${ }^{* *} \mathrm{P}<0.01$ between groups.

attributed to endoplasmic reticulum stress [26]. In this study, we focused on downstream consequences of IH-induced PI3K-AKT inhibition, including GSK3 and FOXO1 signaling, and on physiologic outcomes such as hepatic glycogen synthesis and gluconeogenesis. Moreover, we used a JNK inhibitor to examine roles of JNK activation in the regulation of hepatic insulin signal transduction and glucose metabolism. Our major findings were that 1) IH impaired hepatocyte insulin signal transduction, as evidenced by canonical patterns of AKT/GSK-3 $\beta$ phosphorylation, via JNK activation; and 2) IH decreased FOXO1 expression and gluconeogenesis as assessed by PEPCK transcription, which was independent of insulin signaling. To our knowledge, this is the first in vitro study to show the pleiotropic effects of IH on liver glucose metabolism.

At the cellular signaling level, our results are consistent with the association between OSA and IR. In hepatocytes, insulin stimulates glycogen synthesis via PI3K-AKT phosphorylation, which inactivates GSK-3 and increases glycogen synthase activity [21]. In this study, we revealed an inhibition of insulin-stimulated AKT/GSK-3 $\beta$ phosphorylation in liver cells exposed to IH, indicating hepatic IR. These findings are consistent with the observation that 14 days of IH exposure in mice decreased phosphorylation of liver AKT/GSK-3 $\beta$ [28]. These changes in insulin transduction would be expected to reduce liver glycogen content in response to insulin. The lack of decrease in glycogen content we observed after IH suggests that insulin-independent mechanisms have also contributed to glycogen metabolism in the context of IH.

Furthermore, we observed that IH decreased phosphorylation of FOXO1, which antagonizes the effect of insulin on gluconeogenesis. FOXO1, one of the four FOXO isoforms of forkhead transcription factors, is highly expressed in the liver and its activation regulates the expression of PEPCK and G6Pase, two key gluconeogenic genes, which promote the 
subsequent regulation of glucose output [22]. FOXO1 is regulated by a complex interplay between phosphorylation, acetylation, ubiquitination and other post-translational modifications [22]. Insulin acts through AKT to phosphorylate FOXO1, resulting in its inhibitory nuclear exclusion and consequently inhibiting gluconeogenesis [29]. In this study, we found a parallel inhibition pattern of insulin-stimulated FOXO1 and AKT phosphorylation in liver cells during IH exposure. It is unclear why IH inhibited FOXO1 phosphorylation, yet suppressed transcription of PEPCK. One possibility is that IH also inhibited total FOXO1 expression, which might reduce transcription of PEPCK independent of insulin signaling. The reduced level of FOXO1 may also have contributed towards impaired glycogenolysis [30], leading to glycogen accumulation, which antagonized the effects of decreased glycogen synthesis regulated by AKT/GSK-3 $\beta$ signaling.

A major finding of this study is that JNK activation contributed to IH-induced IR in liver cells. JNKs, members of the mitogen-activated protein kinases (MAPK) family, are implicated in the pathogenesis of IR [23]. JNK activation stimulates serine phosphorylation of IRS-1, which blocks the downstream insulin signal transduction through PI3K-AKT [24, 25]. In the current study, we confirmed phosphorylation and activation of the JNK pathway in $\mathrm{HepG}_{2}$ cells exposed to IH. Moreover, JNK inhibitor (SP600125) pretreatment partially prevented IH-induced inhibition of AKT/GSK-3 $\beta$ phosphorylation in the presence of insulin, and increased glycogen after IH exposure. However, no impact on FOXO1 signaling was observed by SP600125 pretreatment, which further supported our inference that IH inhibited FOXO1 via insulin-independent mechanisms.

In terms of physiologic outcomes such as gluconeogenesis, our results differ from some in vivo studies, illustrating the complex nature of hypoxia on metabolism. We observed that IH suppressed hepatic gluconeogenesis as suggested by lower PEPCK levels and the tendency of decrease in glucose production. By contrast, Polak et al [12] isolated hepatocytes from mice after 14 days of $\mathrm{IH}$ and revealed increased total glucose output as compared to cells from control mice, which was attributed to increased gluconeogenesis. Shin et al [13] also reported increased baseline hepatic glucose output and liver PEPCK transcription in lean mice after 6-week IH exposure. Potentially, the lower PEPCK transcription that we observed from hepatocytes in response to IH does not resemble outcomes from these in vivo experiments because of other systemic effects of IH on catecholamines and hormones, or because of differences in experimental technique. For example, in the study by Polak et al, hepatocytes were cultured in normoxic conditions after isolation for at least $10 \mathrm{~h}$ before the measurements of glucose output; In the study by Shin et al, animals were restrained and anaesthetized throughout the measurement procedure. Interestingly, liver PEPCK transcription was no longer affected by 4-week IH exposure when experiments were performed in unrestrained and unanaesthetized mice [31]. Thus, our experiment provides novel insights into the direct impacts of IH on glucose metabolism in isolated liver cells independent of in vivo neural and endocrine regulation. Our findings challenge the theory that IH in the liver per se impairs glucose homeostasis in OSA patients.

Interpretation of the presented results should respect the limitations of the study. First, this was by design an in vitro study of the isolated metabolic responses of liver cells to IH. In vivo, systemic effects of IH may modify direct hepatic metabolic responses to IH, including autonomic signaling [13], and changes to substrate delivery [32]. Second, as with any in vitro study, there are limitations to the model of IH. Some effects of IH may be explained by activation of anaerobic pathways. For example, the paradoxical trend towards increase in glycogen content after IH, despite inhibition of AKT/GSK-3 $\beta$, may implicate hypoxia inducible factor (HIF)-mediated induction of glycogen synthase 1 [33]. Third, in addition to JNK, changes in other stress/hypoxia-inducible pathways including Sestrins, oxidative stress, as well as microRNA profiles should be considered in interpreting the metabolic consequences of IH in liver cells [34-36].

In summary, the current study demonstrates that IH alters glucose regulation in liver cells, leading to impaired insulin signaling partially mediated by JNK activation, as well as reduced gluconeogenesis. Some of the consequences of IH occur independently of changes 
Gu et al.: Intermittent Hypoxia and Hepatic Glucose Homeostasis

in insulin signaling. In particular, IH inhibition of hepatic FOXO1 may orchestrate reductions in gluconeogenesis and glycogenolysis. We need further investigations to elucidate roles of FOXO1 in regulating metabolism in OSA.

\section{Acknowledgements}

This work was funded by the following grants: National Natural Science Foundation of China (81400065, 81670081, 81070068, 81270148); Key Basic Research Program of Shanghai Science and Technology Commission (11JC1411302); Youth Fund Project of Chinese Sleep Research Society (2014-05); Shanghai Key Discipline for Respiratory Diseases (2017ZZ02014). We thank Prof. Jonathan Jun from Johns Hopkins University for critical reading of this manuscript and his valuable suggestions.

\section{Disclosure Statement}

The authors declare that they have no Disclosure Statement.

\section{References}

1 Shaw JE, Punjabi NM, Wilding JP, Alberti KG, Zimmet PZ: Sleep-disordered breathing and type 2 diabetes: a report from the International Diabetes Federation Taskforce on Epidemiology and Prevention. Diabetes Res Clin Pract 2008;81:2-12.

-2 Pamidi S, Tasali E: Obstructive sleep apnea and type 2 diabetes: is there a link? Front Neurol 2012;3:126.

-3 Punjabi NM, Beamer BA: Alterations in Glucose Disposal in Sleep-disordered Breathing. Am J Respir Crit Care Med 2009;179:235-240.

-4 Pamidi S, Wroblewski K, Broussard J, Day A, Hanlon EC, Abraham V, Tasali E: Obstructive sleep apnea in young lean men: impact on insulin sensitivity and secretion. Diabetes Care 2012;35:2384-2389.

-5 Reinke C, Bevans-Fonti S, Drager LF, Shin MK, Polotsky VY: Effects of different acute hypoxic regimens on tissue oxygen profiles and metabolic outcomes. J Appl Physiol (1985) 2011;111:881-890.

6 Sherwani SI, Aldana C, Usmani S, Adin C, Kotha S, Khan M, Eubank T, Scherer PE, Parinandi N, Magalang UJ: Intermittent hypoxia exacerbates pancreatic beta-cell dysfunction in A mouse model of diabetes mellitus. Sleep 2013;36:1849-1858.

7 Lavie L: Oxidative stress in obstructive sleep apnea and intermittent hypoxia--revisited--the bad ugly and good: implications to the heart and brain. Sleep Med Rev 2015;20:27-45.

8 Nanduri J, Yuan G, Kumar GK, Semenza GL, Prabhakar NR: Transcriptional responses to intermittent hypoxia. Respir Physiol Neurobiol 2008;164:277-281.

9 Bechmann LP, Hannivoort RA, Gerken G, Hotamisligil GS, Trauner M, Canbay A: The interaction of hepatic lipid and glucose metabolism in liver diseases. J Hepatol 2012;56:952-964.

10 Louis M, Punjabi NM: Effects of acute intermittent hypoxia on glucose metabolism in awake healthy volunteers. J.Appl.Physiol 2009;106:1538-1544.

-11 Iiyori N, Alonso LC, Li J, Sanders MH, Garcia-Ocana A, O’Doherty RM, Polotsky VY, O’Donnell CP: Intermittent hypoxia causes insulin resistance in lean mice independent of autonomic activity. Am.J.Respir. Crit Care Med. 2007;175:851-857.

12 Polak J, Shimoda LA, Drager LF, Undem C, McHugh H, Polotsky VY, Punjabi NM: Intermittent hypoxia impairs glucose homeostasis in C57BL6/J mice: partial improvement with cessation of the exposure. Sleep 2013;36:1483-1490; 1490A-1490B.

13 Shin MK, Yao Q Jun JC, Bevans-Fonti S, Yoo DY, Han W, Mesarwi O, Richardson R, Fu YY, Pasricha PJ, Schwartz AR, Shirahata M, Polotsky VY: Carotid body denervation prevents fasting hyperglycemia during chronic intermittent hypoxia. J Appl Physiol (1985) 2014;117:765-776.

14 Wang Q, Sun X, Li X, Dong X, Li P, Zhao L: Resveratrol attenuates intermittent hypoxia-induced insulin resistance in rats: involvement of Sirtuin 1 and the phosphatidylinositol-4, 5-bisphosphate 3-kinase/AKT pathway. Mol Med Rep 2015;11:151-158. 


\section{Cellular Physiology Cell Physiol Biochem 2018;47:1042-1050

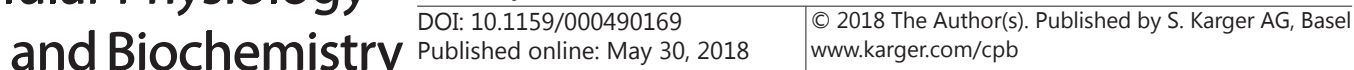

15 Shin MK, Han W, Bevans-Fonti S, Jun JC, Punjabi NM, Polotsky VY: The effect of adrenal medullectomy on metabolic responses to chronic intermittent hypoxia. Respir Physiol Neurobiol 2014;203C:60-67.

16 Girard J: The Inhibitory Effects of Insulin on Hepatic Glucose Production Are Both Direct and Indirect. Diabetes 2006;55:S65-S69.

17 Edgerton DS, Lautz M, Scott M, Everett CA, Stettler KM, Neal DW, Chu CA, Cherrington AD: Insulin's direct effects on the liver dominate the control of hepatic glucose production. J Clin Invest 2006;116:521-527.

-18 Knowles BB, Howe CC, Aden DP: Human hepatocellular carcinoma cell lines secrete the major plasma proteins and hepatitis B surface antigen. Science 1980;209:497-499.

-19 Baker PR, Cramer SD, Kennedy M, Assimos DG, Holmes RP: Glycolate and glyoxylate metabolism in HepG2 cells. Am J Physiol Cell Physiol 2004;287:C1359-1365.

20 Shao J, Qiao L, Janssen RC, Pagliassotti M, Friedman JE: Chronic hyperglycemia enhances PEPCK gene expression and hepatocellular glucose production via elevated liver activating protein/liver inhibitory protein ratio. Diabetes 2005;54:976-984.

21 Saltiel AR, Kahn CR: Insulin signalling and the regulation of glucose and lipid metabolism. Nature 2001;414:799-806.

-22 Eijkelenboom A, Burgering BM: FOXOs: signalling integrators for homeostasis maintenance. Nat Rev Mol Cell Biol 2013;14:83-97.

23 Solinas G, Becattini B: JNK at the crossroad of obesity, insulin resistance, and cell stress response. Mol Metab 2017;6:174-184.

24 Aguirre V, Uchida T, Yenush L, Davis R, White MF: The c-Jun NH(2)-terminal kinase promotes insulin resistance during association with insulin receptor substrate-1 and phosphorylation of Ser(307). J Biol Chem 2000;275:9047-9054.

-25 Aguirre V, Werner ED, Giraud J, Lee YH, Shoelson SE, White MF: Phosphorylation of Ser307 in insulin receptor substrate-1 blocks interactions with the insulin receptor and inhibits insulin action. J Biol Chem 2002;277:1531-1537.

26 Yi H, Gu C, Li M, Zhang Z, Li Q Feng J, Zhou J, Du J: PERK/eIF2alpha contributes to changes of insulin signaling in HepG2 cell induced by intermittent hypoxia. Life Sci 2017;181:17-22.

-27 Lu H, Huang H: FOXO1: a potential target for human diseases. Curr Drug Targets 2011;12:1235-1244.

28 Thomas A, Belaidi E, Moulin S, Horman S, van der Zon GC, Viollet B, Levy P, Bertrand L, Pepin JL, GodinRibuot D, Guigas B: Chronic Intermittent Hypoxia Impairs Insulin Sensitivity but Improves Whole-Body Glucose Tolerance by Activating Skeletal Muscle AMPK. Diabetes 2017;10.2337/db17-0186

29 Petersen MC, Vatner DF, Shulman GI: Regulation of hepatic glucose metabolism in health and disease. Nat Rev Endocrinol 2017;13:572-587.

30 Matsumoto M, Pocai A, Rossetti L, Depinho RA, Accili D: Impaired regulation of hepatic glucose production in mice lacking the forkhead transcription factor Foxo1 in liver. Cell Metab 2007;6:208-216.

-31 Shin MK, Han W, Joo H, Bevans-Fonti S, Shiota M, Stefanovski D, Polotsky VY: Effect of adrenal medullectomy on metabolic responses to chronic intermittent hypoxia in the frequently sampled intravenous glucose tolerance test. J Appl Physiol (1985) 2017;122:767-774.

-32 Gu C, Younas H, Jun JC: Sleep apnea: An overlooked cause of lipotoxicity? Medical Hypotheses 2017;108:161-165.

33 Pescador N, Villar D, Cifuentes D, Garcia-Rocha M, Ortiz-Barahona A, Vazquez S, Ordonez A, Cuevas Y, Saez-Morales D, Garcia-Bermejo ML, Landazuri MO, Guinovart J, del Peso L: Hypoxia promotes glycogen accumulation through hypoxia inducible factor (HIF)-mediated induction of glycogen synthase 1. PLoS One 2010;5:e9644.

34 Wang M, Xu Y, Liu J, Ye J, Yuan W, Jiang H, Wang Z, Jiang H, Wan J: Recent Insights into the Biological Functions of Sestrins in Health and Disease. Cell Physiol Biochem 2017;43:1731-1741.

-35 Zhi F, Shao N, Xue L, Xu Y, Kang X, Yang Y, Xia Y: Characteristic MicroRNA Expression Induced by deltaOpioid Receptor Activation in the Rat Liver Under Prolonged Hypoxia. Cell Physiol Biochem 2017;44:22962309.

-36 Liu Z, Tu K, Wang Y, Yao B, Li Q, Wang L, Dou C, Liu Q, Zheng X: Hypoxia Accelerates Aggressiveness of Hepatocellular Carcinoma Cells Involving Oxidative Stress, Epithelial-Mesenchymal Transition and NonCanonical Hedgehog Signaling. Cell Physiol Biochem 2017;44:1856-1868. 